\title{
A NECESSARY AND SUFFICIENT CONDITION FOR A CONNECTED AMENABLE GROUP TO HAVE POLYNOMIAL GROWTH
}

\author{
S. GANESAN
}

\begin{abstract}
It is shown that a connected amenable group $G$ has polynomial growth if, and only if, given any open subsemigroup $S$ of $G$ and a compact set $K$ in $G$ there exists an $s$ in $S$ such that $K s \subset S$.
\end{abstract}

Introduction. Let $G$ be a connected topological group and $C(G)$ the space of all bounded continuous real-valued functions on $G$. For each $f$ in $C(G)$ and $a$ in $G$ define $\|f\|=\sup \{|f(x)|: x \in G\}$ and $l_{a} f(t)=f\left(a^{-1} t\right)$ for all $t$ in $G$. A function $f$ in $C(G)$ is left uniformly continuous if whenever $\left(s_{i}\right)$ is a net in $G$ and converges to some $s$ in $G$, then $\left\|l_{s_{i}} f-l_{s} f\right\|$ tends to zero. We say that $G$ is amenable (more accurately, left amenable) if there exists an $m$ in $\operatorname{LUC}(G)^{*}$. the conjugate space of $\operatorname{LUC}(G)$ (the space of left uniformly continuous functions), such that $m \geq 0,\|m\|=1$ and $m\left(l_{a} f\right)=m(f)$ for all $f$ in $\operatorname{LUC}(G)$ and $a$ in $G$. The reader is referred to Greenleaf $[\mathbf{3}]$ for many interesting properties of amenable groups. Another interesting class of groups are those with polynomial growth. whose definition follows. Let $|A|_{G}$ denote the left Haar measure of a measurable subset $A$ of $G$. A locally compact group $G$ is said to have polynomial growth if for each compact nbd $U$ of the identity $e$ in $G$. there exists a polynomial $p$ such that $\left|U^{n}\right|_{G} \leq p(n)$ for all $n=1,2,3, \ldots$

The notion of Archimedean property for groups was introduced in [2]. A group $G$ is said to have Archimedean property if whenever $S$ is an open generating subsemigroup and $K$ a compact subset of $G$ there exists an $s$ in $S$ such that $s K \subset S$. It is proved in $[2]$ that any connected group with polynomial growth has Archimedean property. Some remarkably diverse properties of groups with polynomial growth can be found in $[\mathbf{1}, \mathbf{4}, \mathbf{7}]$. It is well known that groups with polynomial growth are amenable. However, the converse fails. The group of affine transformations on the real line is a connected solvable group without polynomial growth. Jenkins [6] has given a necessary and sufficient condition for a connected amenable group to have polynomial growth. In this paper we give another necessary and sufficient condition in Theorem 2. As a corollary we note that every bounded continuous function on a group with polynomial growth which is left uniformly continuous on a dense open subsemigroup is left uniformly continuous on $G$.

First we prove the following

Proposition 1. Let $G$ be a connected locally compact group and $S$ an open subsemigroup of $G$. Then the following are equivalent.

Received by the editors October 31, 1983 and, in revised form, January 4, 1984.

1980 Mathematics Subject Classification. Primary 22E15, 22A05; Secondary 20M05.

Key words and phrases. Polynomial growth, Archimedean property, Amenable group, subsemigroup. 
(a) The open right ideals of $S$ have finite intersection property, i.e., any finite set of nonempty open right ideals of $S$ has nonempty intersection.

(b) $G=S S^{-1}$.

(c) Given any compact set $K$ in $G$ there exists an $s$ in $S$ such that $K s \subseteq S$.

Proof. (b) implies (c). First, we prove that given any finite subset $F$ of $G$ there exists a $t$ in $S$ such that $F t \subseteq S$. If $F=\left\{k_{1}, k_{2}, \ldots, k_{n}\right\}$ there exists an $s_{1}$ in $S$ such that $k_{1} s_{1}$ is in $S$; there exists an $s_{2}$ in $S$ with $\left(k_{2} s_{1}\right)$ in $S$, etc. Then $F s_{1} s_{2} \cdots s_{n}$ is a subset of $S$. Now, if $K$ is any compact subset (given) fix an $s$ in $S$. Let $U=U^{-1}$ be a nbd of $e$ with compact closure such that $A=s U$ is a subset of $S$. Consider the compact set $s^{-1} K$; since $G$ is connected, there is a large $N$ such that $s^{-1} K \subset U^{N}$. Since $U$ is an open set with compact closure there exists a finite set $F$ such that $U U \subset \overline{U U} \subset U F$ and, choosing $t$ as above, $U^{N} t \subset U F^{N-1} t \subset U S$. Hence, $K t \subset s U^{N} t \subset s U S \subset S S \subset S$, which proves (c).

The proof of (c) implies (b) is obvious.

(b) implies (a). Suppose $I$ and $J$ are two open right ideals of $S$ which are disjoint. Consider $b^{-1} a$ in $G$ where $a \in I, b \in J$; then $b^{-1} a=s_{1} s_{2}^{-1}$ for some $s_{1}$ and $s_{2}$ in $S$. Hence, $\left(b s_{1}\right)^{-1}=s_{1}^{-1} b^{-1}=s_{2}^{-1} a^{-1}=\left(a s_{2}\right)^{-1}$, so that $I^{-1} \cap J^{-1} \neq \varnothing$, a contradiction.

(a) implies (b). $S S^{-1}$ is an open subgroup of $G$ containing $e$, the identity of $G$. For, if $a, b, c$ and $d$ are in $S$ then $a b^{-1} c d^{-1} \in S S^{-1}$. Since $c S$ and $b S$ are open right ideals in $S, c S$ and $b S$ have an element in common, i.e., there exists $s_{1}, s_{2}$ in $S$ such that $c s_{1}=b s_{2}$, which implies $a b^{-1} c d^{-1}=a s_{2}\left(d s_{1}\right)^{-1} \in S S^{-1}$. This shows that $S S^{-1}$ is an open subgroup and hence closed. Since $G$ is connected, $G=S S^{-1}$.

With very few modifications to [2, Lemma 7] one can show that any connected locally compact group with polynomial growth has property (c) of the above proposition. Also, note that $G=S^{-1} S$ for any open subsemigroup $S$ of $G$ is equivalent to: given any compact set $K$ in $G$ there exists an $s$ in $S$ such that $s K \subset S$. The above two remarks imply that if $G$ has polynomial growth then for any open subsemigroup $S$ of $G, G=S S^{-1}=S^{-1} S$.

Jenkins $[\mathbf{5}, \mathbf{6}]$ has shown that a connected amenable group $G$ has polynomial growth if, and only if, each open subsemigroup of $G$ is amenable if, and only if, the open right ideals of an open subsemigroup of $G$ have finite intersection property. Using the above remark and the Proposition we have

THEOREM 2. Let $G$ be a connected group. Then the following are equivalent.

(a) $G$ is an amenable group with the property that given any compact set $K$ in $G$ and any open semigroup $S$ of $G$, then there exists an $s$ in $S$ such that $K s \subset S$.

(b) $G$ has polynomial growth.

The following is worth noting.

COROLLARY. Let $G$ be a connected group with polynomial growth and $S$ an open dense subsemigroup of $G$. If $F$ is a bounded continuous function on $G$ such that $F_{\mid S}$ is in $\operatorname{LUC}(S)$, then $F$ is in $\operatorname{LUC}(G)$.

Proof. Since $G$ has polynomial growth, it is an Archimedean group and $G=$ $S S^{-1}$. The rest of the proof follows from Lau [8].

ACKNOWLEDGEMENT. The author would like to thank the referee for suggestions and corrections he made on the original manuscript. 
The author also wishes to thank Professor Joe Jenkins for helpful discussions related to this paper.

\section{REFERENCES}

1. J. Dixmier, Opérateurs de rang fini dans les représentations unitaires, Inst. Hautes Études Sci. Publ. Math. 6 (1960), 305-317.

2. S. Ganesan and J. W. Jenkins, An Archimedean property for groups with polymomial growth. Proc. Amer. Math. Soc. 88 (1983), 550-554.

3. F. P. Greenleaf, Invariant means on topological groups and their applications, Vail Nostrand. New York, 1969.

4. A. Hulanicki, Subalgebra of $L^{1}(G)$ associated with Laplacian on a Lie group. Colloq. Math. 31 (1974), 259-289.

5. J. W. Jenkins, Amenable subsemigroups of a locally compact group, Proc. Amer. Math. Soc. 25 (1970), 766-770.

6. __ Følner's condition for exponentially bounded groups, Math. Scand. 35 (1974), 165174

7. __ Representations of exponentially bounded groups, Amer. J. Math. 98 (1976), 2938.

8. A. T. Lau, Invariant means on dense subsemigroups of topological groups, Canad. J. Math. 23 (1971), 797-801.

Department of Mathematics, University of Portland, Portland, Oregon 97203 